\title{
Vagus Nerve: The Key Integrator of Anti-inflammatory Reflex
}

\author{
Gopal Krushna Pall ${ }^{1,}$, Nivedita Nanda ${ }^{2}$
}

\section{Gopal Krushna Palli,*, Nivedita Nanda ${ }^{2}$}

\author{
'Editor-in-Chief, IJCEP. \\ ${ }^{2}$ Editor, Experimental Physiology \\ Section, IJCEP. \\ Correspondence \\ Dr. Gopal Krushna Pal \\ Editor-in-Chief, IJCEP. \\ Phone: +91-9344291160 \\ Email: drgkpa|@gmail.com \\ History \\ - Submission Date: 12-12-2019; \\ - Review completed: 17-02-2020; \\ - Accepted Date: 02-03-2020.
}

DOI : 10.5530/ijcep.2020.7.1.1

Article Available online

http://www.ijcep.org

\section{Copyright}

(c) 2020 Phcog.Net. This is an openaccess article distributed under the terms of the Creative Commons Attribution 4.0 International license.
Inflammation is a local response of tissue to microbial invasion or injury, which is usually a protective phenomenon. This inflammatory response, also called as inflammatory reflex, should be regulated precisely as deficiency or excess of the inflammatory response can cause morbidity and reduce lifespan. ${ }^{[1]}$ If inflammation spreads into the bloodstream, as occurs in septic shock syndrome, systemic sepsis, meningitis and severe trauma, the inflammatory responses can be more harmful. Homeostasis is restored when inflammation is limited by antiinflammatory responses that are adaptive to changes in input and integration by autonomic nervous system (ANS). The recent discovery that cholinergic neurons inhibit acute inflammation has facilitated our understanding of how the ANS moderates immune responses. Recent studies have identified a basic neural pathway that reflexively monitors and inhibits inflammatory response. Therefore, the activation of anti-inflammatory responses has collectively been termed as anti-inflammatory reflex. Inflammatory stimuli activate sensory pathways that relay information to the hypothalamus, which integrates and mobilizes defences to eliminate the inflammation.

The inflammation-sensing and inflammationmediating functions are the principal components of the inflammatory reflex. Though, the inflammation is mediated by many chemicals, important among them are tumor necrosis factor (TNF), IL-1 and high-mobility group B1 (HMGB1). ${ }^{[2]}$ These inflammatory mediators are potentially harmful. The anti-inflammatory reflex counters the effects of the inflammatory chemicals by increasing the production of anti-inflammatory chemicals. Counter-regulatory mechanisms that are highly conserved limit the acute inflammatory response and prevent the spread of inflammatory mediators into the bloodstream. Activated immunologically competent cells release TNF receptor fragments that bind and neutralize its inflammatory and potentially toxic actions. Antiinflammatory cytokines, such as IL-10 and transforming growth factor- $\beta$ (TGF- $\beta$ ), specifically inhibit the release of TNF and other proinflammatory mediators. ${ }^{[3]}$ Adrenal glucocorticoids, adrenaline, $\alpha$-melanocyte-stimulating hormone ( $\alpha-\mathrm{MSH})$ and other 'classical' stress hormones inhibit cytokine synthesis and intracellular signal transduction. ${ }^{[4,5]}$ In the absence of an adequate adrenocorticotropic hormone (ACTH) and glucocorticoid response, TNF is significantly overexpressed during endotoxic systemic inflammation. In animal models, it has been noted that deficiency of IL-10 leads to inflammatory bowel disease and severe form of collageninduced arthritis. ${ }^{[6]}$ These reports indicate that loss of endogenous anti-inflammatory mechanisms converts a normally protective, self-limited inflammatory response into an excessive, potentially deleterious response.

Recently, as the basic reflex pathway that regulates inflammation has been identified, documentation has been made of a neural anti-inflammatory mechanism that inhibits macrophage activation through parasympathetic system. ${ }^{[7]}$ This system has been designated as the 'cholinergic anti-inflammatory pathway' because acetylcholine is the principle parasympathetic neurotransmitter released in this reflex pathway, and macrophages that are exposed to acetylcholine are effectively deactivated. ${ }^{[8]}$ The vagus nerve innervates many visceral organs, including those that contain the reticuloendothelial system (liver, lung, spleen, kidneys and gut). Experimental activation of the cholinergic anti-inflammatory pathway by direct electrical stimulation of the efferent vagus nerve inhibits the synthesis of TNF in liver, spleen and heart, and attenuates serum concentrations of TNF during endotoxemia. ${ }^{[7]}$ Vagotomy significantly exacerbates TNF responses to inflammatory stimuli and sensitizes animals to the lethal effects of endotoxin. Interaction between the macrophage cholinergic receptor and its ligand inhibits the synthesis of proinflammatory cytokines (TNF, IL-1 and IL-18) but not anti-inflammatory cytokines (such as IL-10). ${ }^{[7]}$ Stimulation of efferent vagus nerve activity has been associated classically with slowing of heart rate, induction of gastric motility, dilation of arterioles, constriction of pupils and as recently proposed the inhibition of the inflammatory responses. The vagal anti-inflammatory network that includes release of glucocorticoids, anti-inflammatory cytokines and other humoral mediators of inflammation, is distributed to many tissues of the body and its subcomponent the cholinergic anti-inflammatory pathway is discrete and localized in tissues. Thus, vagal anti-inflammatory responses have both systemic and local responses.

The anti-inflammatory reflex mechanisms of vagus nerve are mediated through several pathways.

- The first one is the anti-inflammatory hypothalamic-pituitary-adrenal axis which is stimulated by vagal afferent fibres and leads to the release of cortisol by the adrenal glands. ${ }^{[9]}$ Many systemic

Cite this article: Pal GK, Nanda N. Vagus nerve: the key integrator of anti-inflammatory reflex. Int J Clin Exp Physiol. 2020;7(1):1-2. 
effects occur because vagus nerve activity relays to the medullary reticular formation, locus coeruleus and hypothalamus, and activation of these structures leads to increased release of ACTH from the anterior pituitary.

- The second one, called the cholinergic anti-inflammatory pathway, is mediated through vagal efferent fibres that synapse onto enteric neurons which release acetylcholine $(\mathrm{ACh})$ at the synaptic junction with macrophages. ${ }^{[9]} \mathrm{ACh}$ binds to $\alpha$-7-nicotinic ACh receptors of those macrophages to inhibit the release of tumour necrosis factor alpha (TNF- $\alpha$ ), a pro-inflammatory cytokine.

- The third pathway is the splenic sympathetic anti-inflammatory pathway, where vagus nerve stimulates the splenic sympathetic nerve. ${ }^{[9]}$ Noradrenaline released at the distal end of the splenic nerve links to the $\beta_{2}$ adrenergic receptor of splenic lymphocytes that release ACh.

- Finally, ACh inhibits the release of TNF- $\alpha$ by spleen macrophages through $\alpha$-7-nicotinic ACh receptors. ${ }^{[9]}$

Understanding of these components of vagal anti-inflammatory reflex pathways has therapeutic implications, since they could be targeted in various means to stimulate anti-inflammatory regulation in $\operatorname{TNF} \alpha$ related diseases such as inflammatory bowel disease and rheumatoid arthritis. The vagal nerve stimulation, either as an invasive or noninvasive procedure, is becoming increasingly the objective of many researches to evaluate the potential effectiveness of vagus nerve stimulation (VNS) to alleviate chronic inflammation. As such VNS has recently been attempted in the management of many clinical disorders. ${ }^{[10]}$ It has been suggested if pranayamas as part of the yogic practices can yield the effects of VNS. ${ }^{[11]}$ Currently, inflammation has been found to be an important etiopathogenic mechanism in the causation of many metabolic disorders. Thus, role of vagus nerve as the key integrator of antiinflammatory reflex and the efficacy of VNS as a therapeutic tool will be the target of many future clinical researches in finding-out a promising solution to the management of lifestyle diseases.

\section{REFERENCES}

1. Tracey KJ. The inflammatory reflex. Nature. 2002;420:853-59.

2. Wang $H$, Yang H, Czura CJ, Sama AE, Tracey KJ. HMGB1 as a late mediator of lethal systemic inflammation. Am J Respir Crit Care Med. 2001;164(10):1768-73.

3. Tsunawaki S, Sporn M, Ding A, Nathan C. Deactivation of macrophages by transforming growth factor-b. Nature. 1998;334(6179):260-2

4. Scheinman RI, Cogswell PC, Lofquist AK, Baldwin AS. Role of transcriptional activation of $\mathrm{IkBa}$ in mediation of immunosuppression by glucocorticoids. Science. 1995;270(5234):283-6.

5. Chrousos GP. The stress response and immune function: clinical implications. The 1999 Novera H. Spector Lecture. Ann NY Acad Sci. 2000;917(8):38-67.

6. Johansson AC, Hansson AS, Nandakumar KS, Backlund J, Holmdahl R. IL-10deficient B10. Q mice develop more severe collagen-induced arthritis, but are protected from arthritis induced with anti-type II collagen antibodies. J Immunol. $2001 ; 167(6): 3505-12$.

7. Borovikova LV, Ivanova S, Zhang M, Yang H, Botchkina Gl, Watkins LR, et al. Vagus nerve stimulation attenuates the systemic inflammatory response to endotoxin. Nature. 2000;405(6785):458-62.

8. Hermann GE, Emch GS, Tovar CA, Rogers RC. c-Fos generation in the dorsal vagal complex after systemic endotoxin is not dependent on the vagus nerve. Am J Physiol Regul Integr Comp Physiol. 2001;280(1):R289-99.

9. Bonaz B, Sinniger V, Pellissier S. Anti-inflammatory properties of the vagus nerve: potential therapeutic implications of vagus nerve stimulation. J Physiol. 2016;594(20):5781-90

10. Johnson $R L$, Wilson $C G$. A review of vagus nerve stimulation as a therapeutic intervention. J Inflamm Res. 2018;11:203-13.

11. Pal GK. Pranayama could be the best nonpharmacological and nonsurgical method of vagal nerve stimulation. Int J Clin Exp Physiol 2018;5(2):59-60.

Cite this article: Pal GK, Nanda N. Vagus nerve: the key integrator of anti-inflammatory reflex. Int J Clin Exp Physiol. 2020;7(1):1-2. 